\title{
Design and Completion of the Indoor Positioning System Based-on Taylor Iterative
}

\author{
Liyan FAN ${ }^{1, a, ~}{ }^{\text {, }}$, Song $\mathrm{CHEN}^{2, \mathrm{~b}}$, Shuang WAN ${ }^{3, \mathrm{c}}$, Shilei ZHU ${ }^{4}$ \\ ${ }^{1}$ Institute of Navigation and Space Target Engineering, Information Engineering University, \\ Zhengzhou Henan, 450001, China \\ ${ }^{2}$ Institute of Navigation and Space Target Engineering, Information Engineering University, \\ Zhengzhou Henan, 450001, China \\ ${ }^{3}$ Institute of Information Science and Engineering, Southwest Jiaotong University, \\ Chengdu Sichuan, 611756, China \\ ${ }^{4}$ Institute of Navigation and Space Target Engineering, Information Engineering University, \\ Zhengzhou Henan, 450001, China \\ aemail:13017653962@163.com, bemail:wirelessmancs@163.com \\ `email:1051923729@qq.com, ${ }^{*}$ corresponding author
}

Keywords: LBS, Indoor Location System, CSS, WSN, Taylor Iterative Algorithm

\begin{abstract}
Development belonging to Location-Based Service (LBS) makes people's daily lives more convenience. Compared with Outdoor Location System, which developing more and more mature, Indoor Location System achieves much more attention from community. In this paper, by comparing the advantages and disadvantages of existing Indoor Location System comprehensively, the Indoor Wireless Sensor Network (WSN) Positioning system based on Chirp Spread Spectrum (CSS) is chosen as studying target. Taylor iterative algorithm, which enhances the positioning accuracy effectively, is applied to calculate position coordinates.
\end{abstract}

\section{Introduction}

With the popularity of wireless networks, Location-Based Service has achieved more and more attention showing great vitality in emergency relief, transportation, police and fire departments, personalized service and other areas ${ }^{[1]}$. According to the institution predicted, Global LBS market would grow rapidly at the rate of $80 \%$ per year. Visible, LBS deserves to be studied.

At present, the Indoor Navigation System is not yet mature because of a variety of complex technical systems. So how to break though the problems of Indoor Positioning field will be a hotspot. The paper [2] introduces the key technologies of Indoor Positioning based on WLAN with two main positioning method, like paper [3] mentions fingerprint localization algorithm having relatively high positioning accuracy and paper [4] mentions a kind of Indoor Position based on received signal strength indicator (RSSI) though filter algorithm and iterative algorithm; the paper [5] mentions a technology about Indoor Positioning based on Bluetooth 4.0 to solve the traditional problem of high power consumption of Bluetooth devices. Because of high cost and intensive nodes, this algorithm whose positioning accuracy is $3 \mathrm{~m}$, is limited to popularize in many fields; the paper [6] mentions a technology about Indoor Positioning based on Ultra-Wide Band (UWB) with a high accuracy at centimeters. Similarly, it can't be popularized because of high hard-ware cost and algorithm complexity.

After a comprehensive comparative analysis, this paper adopts a modified WSN based on CSS signal Indoor Positioning technology ${ }^{[7]}$ with accuracy less $1 \mathrm{~m}$. In a range of $100 \mathrm{~m} * 100 \mathrm{~m}$, four stations are needed justly to complete position, which reduces hard-ware cost largely. The simulation proves that positioning accuracy can be enhanced further though adopting Taylor iteration. 


\section{Positioning Principle}

Generally, the location system obtains the measured distance by estimating TOA ${ }^{[8]}$ and multiplied by signal transmission speed. In order to making sure the result's veracity, the time of transmiting terminal and receiving terminal is asked to be synchronous strictly in this process, which leads to the difficulty in laying synchronizing network, high cost and inadequacy faceing to popularity. Because of these disadvantages, we promote an asynchronous measurement method ${ }^{[9]}$, namely bilateral TOA estimation method. The steps are as follows.

1) Positioning Label sends ranging request to Measuring Station and marks the time as $t_{11}$;

2) Measuring Stations mark the time as $t_{21}$ and produces responses ACK after receiving the request from Positioning Label;

3) Measuring Stations send ACK, mark the time as $t_{22}$, and calculate $T 2=t_{22}-t_{21}$;

4) Positioning Label marks the time as $t_{12}$ and calculate $T 1=t_{12}-t_{11}$ after receiving ACK;

5) Measuring Stations launch the same measuring distance flow and calculate $T 3$, $T 4$, where $T 3=t_{24}-t_{23}, T 4=t_{14}-t_{13}$

6) Positioning Label calculates the TOA as follows:

$T=\frac{T 1-T 2+T 3-T 4}{4}=\frac{T 1-T 4+T 3-T 2}{4}$

Then completing measuring distance.

This measuring method doesn't need synchronization mechanism, eliminates error caused by clock, and promotes the measuring accuracy.

\section{Location solver principle}

The Positioning Control Center can calculate label's coordinate by combining the distance between the Positioning Label and the Measuring Stations with the Measuring Stations' coordinates. The concrete principle is as follows.

Setting the Positioning Label and the Measuring Stations' coordinates as $(x, y),\left(x_{i}, y_{i}\right)$, let $f_{i}(\mathrm{x}, \mathrm{y})$ as:

$$
f_{i}(\mathrm{x}, \mathrm{y})=\sqrt{\left(\mathrm{x}_{i}-\mathrm{x}\right)^{2}+\left(\mathrm{y}_{i}-\mathrm{y}\right)^{2}}
$$

where $i=1,2, \cdots, N, N$ is the number of stations. Ignore the second and higher components, After spreading the coordinate $\left(x^{n}, y^{n}\right)$ according to Taylor, we can receive

$f_{i}(x, y)=f_{i}^{n}\left(x^{n}, y^{n}\right)+\left.\frac{\partial f(x, y)}{\partial x}\right|_{\left(x^{n}, y^{n}\right)} \delta_{x}+\left.\frac{\partial f(x, y)}{\partial y}\right|_{\left(x^{n}, y^{n}\right)} \delta_{y}$

where $f_{i}^{n}\left(x^{n}, y^{n}\right)=\sqrt{\left(\mathrm{x}_{i}-\mathrm{x}^{n}\right)^{2}+\left(\mathrm{y}_{i}-\mathrm{y}^{n}\right)^{2}}$, then

$$
f_{i}(x, y) \approx f_{i}^{n}\left(x^{n}, y^{n}\right)+e_{x}^{n} \square x+e_{x}^{n} \square y
$$

where $e_{x^{i}}^{n}=\frac{x-x^{i}}{f_{i}^{n}\left(x^{n}, y^{n}\right)}, \quad e_{y^{i}}^{n}=\frac{y-y^{i}}{f_{i}^{n}\left(x^{n}, y^{n}\right)}$, now

$$
\left[\begin{array}{l}
f_{1}(x, y)-f_{1}^{n}\left(x^{n}, y^{n}\right) \\
f_{2}(x, y)-f_{2}^{n}\left(x^{n}, y^{n}\right) \\
f_{3}(x, y)-f_{3}^{n}\left(x^{n}, y^{n}\right) \\
f_{4}(x, y)-f_{4}^{n}\left(x^{n}, y^{n}\right)
\end{array}\right]=\left[\begin{array}{cc}
e_{x^{1}}^{n} & e_{y^{1}}^{n} \\
e_{x^{2}}^{n} & e_{y^{2}}^{n} \\
e_{x^{3}}^{n} & e_{y^{3}}^{n} \\
e_{x^{4}}^{n} & e_{y^{4}}^{n}
\end{array}\right]\left[\begin{array}{l}
\Delta x \\
\Delta y
\end{array}\right]
$$

\section{Let}




$$
\boldsymbol{L}^{n}=\left[\begin{array}{l}
f_{1}(x, y)-f_{1}^{n}\left(x^{n}, y^{n}\right) \\
f_{2}(x, y)-f_{2}^{n}\left(x^{n}, y^{n}\right) \\
f_{3}(x, y)-f_{3}^{n}\left(x^{n}, y^{n}\right) \\
f_{4}(x, y)-f_{4}^{n}\left(x^{n}, y^{n}\right)
\end{array}\right], \quad \boldsymbol{E}=\left[\begin{array}{cc}
e_{x^{1}}^{n} & e_{y^{1}}^{n} \\
e_{x^{2}}^{n} & e_{y^{2}}^{n} \\
e_{x^{3}}^{n} & e_{y^{3}}^{n} \\
e_{x^{4}}^{n} & e_{y^{4}}^{n}
\end{array}\right], \quad \boldsymbol{D}=\left[\begin{array}{c}
\Delta x \\
\Delta y
\end{array}\right]
$$

Then $\boldsymbol{L}^{n}=\boldsymbol{E} \boldsymbol{D}$ is a group of overdetermination equation, which can be solved by the Least Squares:

$$
D=\left(E^{T} E\right)^{T} E^{T} L^{n}
$$

Then, the coordinate $\left(x^{n+1}, y^{n+1}\right)$ is

$$
\left[\begin{array}{l}
x^{n+1} \\
y^{n+1}
\end{array}\right]=\left[\begin{array}{l}
x^{n} \\
y^{n}
\end{array}\right]+\boldsymbol{D}
$$

Repeat the above steps, if $\Delta x, \Delta y$ reach the preset threshold, namely $\square x+\square y<\xi(\xi>0)$, the current coordinate $\left(x^{*}, y^{*}\right)$ is the final label's coordinate.

\section{Positioning Accuracy Analysis}

In order to testing the positioning accuracy of the system, the position of the measuring stations need to be calibrated firstly. Trimble S8 Total Station (shown below), which has an angle measurement accuracy of \pm 1 " and ranging accuracy of $\pm(3 \mathrm{~mm}+2 \mathrm{ppm})$ under the direct reflection mode, is used to calibrate the measuring stations. In the test, a room with $3.5 \mathrm{~m}$ in height, $12 \mathrm{~m}$ in length and $7.2 \mathrm{~m}$ in width was chosen, and four measuring stations were hung in four corners of it. Nine labels were placed in nine random chosen positions, and being measured respectively by using the proposed Indoor Location System and total station. Though contrasting between the measured data and real data, the statistical cumulative error curve of the positioning system is obtained as follows:

As can be seen from the figure, the probability of the positioning error less than $0.8 \mathrm{~m}$ can reach $90 \%$, so a conclusion that the positioning accuracy of the positioning system is better than $1 \mathrm{~m}$ can be got.

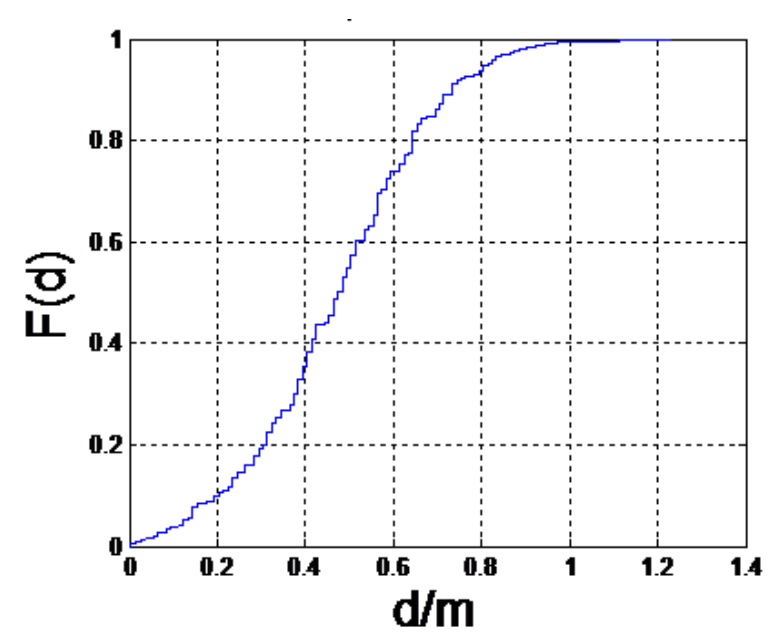

Fig 2.8. Cumulative error curve

\section{Test results}

Plan a rectangular path with a width of $4.8 \mathrm{~m}$ and $9.6 \mathrm{~m}$ in length in the room above, hold the poisoning label and move along the path with a speed at about $0.8 \mathrm{~m} / \mathrm{s}$ adopting the mentioned CSS 
indoor positioning technology to locate, then calculate the original position by using the maximum likelihood algorithm. The original result and the filtered result though Kalman are shown below:

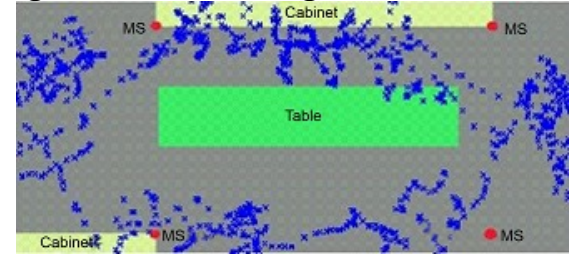

(a) the maximum likelihood algorithm

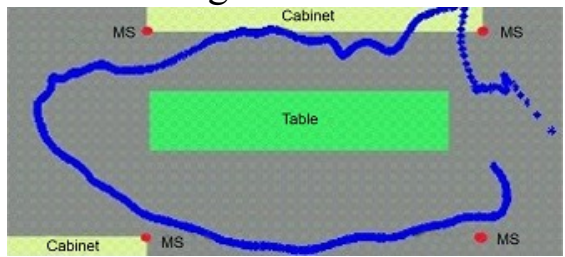

(b) Maximum likelihood +Kalman

Fig 3.1. Original result and filtered result

The calculated result of the Taylor iteration algorithm and The filtered result though Kalman are shown as below.

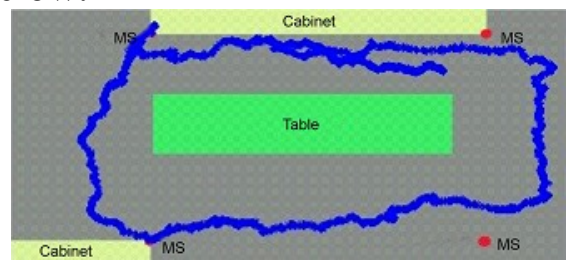

(a) Taylor iteration algorithm

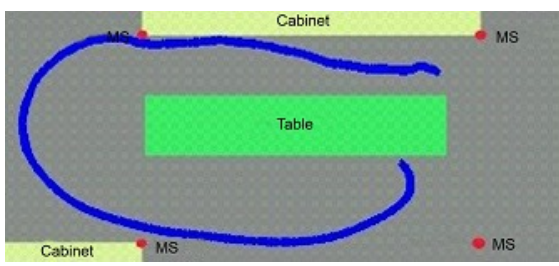

(b) Taylor iteration + Kalman

Fig 3.2. Result of Taylor iteration algorithm and filtered result

\section{Conclusion}

As can be seen from the above test results, the solving effect of the positioning coordinate based on Taylor iteration is much better than solutions based on maximum likelihood algorithm; the smoothness of the positioning trajectory has been significantly improved after adding the Kalman filter, which also leaded to a serious information loss and increased the complexity of the algorithm. Therefore, although we use Taylor iterative algorithm based solution to ensure the positioning accuracy and projects implementability in this paper, the areas need further improvement is needed in the respect of track smoothen, which will be part of the content of the next research.

\section{References}

[1] L L Zhang, E S Zhong. Proceedings of the 8th China Association of GIS Annual Meeting [C]. 2014, 20(11):974-980

[2] Yang Q, Chen Y, Yin J, et al., LEAPS: A Location Estimation and Action Prediction System in a Wireless LAN Environment. In Proceedings of NPC. LNCS 3222,Wuhan, China: 2004,584-591

[3] Bah P, Padmanabhan V N. RADAR: An In-Building RF-Based User Location and Tracking System[C]. 19th IEEE Computer and Communications Societies Conference, Tel Aviv, Israel: IEEE. 2000: $775-784$

[4] J Wang, H Q Zhang. Research on RSSI-based location Technology for Indoor Environments [A]. Computer Measurement and Control, 2009, 17(12): 2500-2503

[5] H S Bian. Research of Indoor Location Technology Based on Bluetooth Low Energy 4.0[D]. Master degree theses. Beijing university of posts and telecommunications. 2015:

[6] Zhongjuan Zhang. Study on Indoor Location Technology Based on UWB [D]. Tianjin: Master's degree thesis of Tianjin University. 2011:

[7] Jianping Luo, Yiping Liu, Mianhe Xiao. Real Time Location System Based on CSS technologyd [J]. SCIENCE \& TECHNOLOGY INFORMATION. 2014, 03(01): 5-7

[8] Bingbing QI, Changqi WU. TOA estimated by combining matched filter and cross correlation [J]. Audio Engineering. 2013, 10(11): 63-65 
[9] Fangxiao Chen. Research and Implementation on UWB Indoor Positioning System Based on synchronous TOA Estimation [D]. Haerbin: Master's degree thesis of Harbin Institute of Technology. 2012.12: 